\title{
Correlation energy contribution to nuclear masses
}

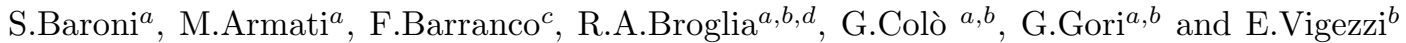 \\ a Dipartimento di Fisica, Università degli Studi di Milano,via Celoria 16, 20133 Milano, Italy. \\ ${ }^{b}$ INFN, Sezione di Milano, via Celoria 16, 20133 Milano, Italy. \\ c Departamento de Fisica Aplicada III, Escuela Superior de Ingenieros, \\ camino de los Descubrimientos s/n, 41029 Sevilla, Spain. \\ ${ }^{d}$ The Niels Bohr Institute, University of Copenhagen, Blegdamsvej 17, 2100 Copenhagen Ø, Denmark.
}

(Dated: July 18, 2018)

\begin{abstract}
The ground state correlation energies associated with collective surface and pairing vibrations are calculated for $\mathrm{Pb}$ - and $\mathrm{Ca}$-isotopes. It is shown that this contribution, when added to those predicted by one of the most accurate modern nuclear mass formula (HFBCS MSk7 mass formula), reduces the associated rms error by an important factor, making mean field theory, once its time dependence is taken into account, a quantitative predictive tool for nuclear masses.

PACS numbers:
\end{abstract}

Many of the nuclei in atoms we find in nature are processed in the stars. In particular, heavy elements far beyond ${ }^{56} \mathrm{Fe}$ are formed through a chain of neutron capture reactions with subsequent beta decay. The source of the rather low energy neutrons are reactions like $(\alpha, \mathrm{n})$ on ${ }^{22} \mathrm{Ni}$ and ${ }^{13} \mathrm{C}$ where the alphas are highly abundant during He burning in the stars. The small neutron abundance then causes a particular path to be followed which lies mainly along the valley of beta stability as the capture process follows a time scale controlled by the variety of $\beta$-decay processes. The end point of this slow processes (s-process) is above ${ }^{209} B i$ via $\alpha$-decay. There is clear evidence for sharp peaks in the abundance curve near neutron closed shell configurations which commonly result from the existence of slow neutron capture processes. However, the abundance curve displays a number of additional peaks at around 8-12 mass units prior to the s-process abundance peaks. This indicates that other capture processes where neutron capture is involved have to take place to produce the corresponding elements as well as elements beyond ${ }^{209} \mathrm{Bi}$. This is called the rapid or r-process. It is known that the r-process path evolves along the neutron drip line region [1], where the binding energy of the least bound neutron is quite small (few hundred $\mathrm{KeV}$ ) in the presence of a large neutron flux 2]. The need for a formula able to predict known masses with an accuracy of this order of magnitude seems quite natural. On the other there still exist many uncertainties in extrapolating our present-day knowledge of formulas which predict nuclear masses, towards the region of the drip line where weakly bound nuclei are found. In fact, the best account of the experimental data based on mean-field theory provides a fitting to the 2135 measured masses with N,Z $>8$ with a rms error of $0.674 \mathrm{MeV}$ 3]. This has been achieved by means of Hartree-FockBardeen-Cooper-Schrieffer (HFBCS) calculations which employ a Skyrme-type zero-range effective force in the mean field channel, supplemented by a zero-range pairing interaction. The 14 parameters set is named BSk2. As a reference point for our work, we have considered a parameter set of almost equal quality, denoted by MSk7, where the rms error is $0.738 \mathrm{MeV}$ [4]. Although this accuracy is remarkable, one is still not satisfied. In fact, while it takes tens of $\mathrm{MeV}$ to remove two nucleons from a nucleus lying along the valley of stability, this quantity becomes as low as $300 \mathrm{keV}$ in halo nuclei like ${ }^{11} \mathrm{Li}$ [5]. The need for a mass formula at least a factor of two more accurate than the MSk7 mass formula is evident.

Nuclei display both single-particle and collective degrees of freedom. Consequently, the corresponding ground states and associated nuclear masses reflect the zero point fluctuations (ZPF) associated with these modes. While mean field theory includes fluctuations associated with quasiparticles, it is only time dependent mean field theory which takes into account the zero point fluctuations associated with collective modes. Despite this, the information in the literature concerning ground state correlation energies associated with collective modes is rather scarce. A recent suggestion to consider their effect was put forward by G.F. Bertsch and K. Hagino [6]. Later, realistic calculations for the quadrupole degree of freedom were performed for light nuclei [ ] and for a few selected isotopes within the socalled Generator Coordinate Method (GCM) [8].

In the present paper we work out, making use of Random Phase Approximation (RPA) calculations, the ground state correlation energies associated with both surface (quadrupole and octupole modes) and pairing vibrations for the $\mathrm{Ca}$ - and $\mathrm{Pb}$-isotopes. Because pairing vibrations [9] have a collective character only around closed shell nuclei (being essentially pure twoquasiparticle states lying on top of twice the pairing gap in superfluid systems, cf. also [10]), one expects the associated ZPF to lead to important corrections to the mass formula [3]. This in keeping with the fact that the largest deviation from experiment found in this mass formula are observed in closed shell systems. It will be concluded that $\mathrm{ZPF}$ are important, in particular those associated with pairing vibrations in closed shell nuclei, in reducing the rms of the Skyrme-HFBCS mass formulas.

To derive the particle-hole RPA equations use can be made of the quasi-boson approximation where the 
particle-hole operators $a_{k}^{+} a_{i}$ and $\left(a_{k}^{+} a_{i}\right)^{+}$are replaced by the boson operators $\Gamma_{k i}^{+}$and $\Gamma_{k i}$. Because collective vibration can be viewed as correlated particle-hole excitations, the corresponding boson creation operator can be written as

$$
\Gamma_{\alpha}^{+}(n)=\sum_{k i}\left(X_{k i}^{\alpha}(n) \Gamma_{k i}^{+}+Y_{k i}^{\alpha}(n) \Gamma_{k i}\right),
$$

where $X_{k i}^{\alpha}$ and $Y_{k i}^{\alpha}$ are the forwardsgoing and backwardsgoing amplitudes fullfilling the normalization condition

$$
\left[\Gamma_{\alpha}(n), \Gamma_{\alpha}^{+}(n)\right]=\sum_{k i}\left(\left|X_{k i}^{\alpha}\right|^{2}-\left|Y_{k i}^{\alpha}\right|^{2}\right)=1 .
$$

The equations which determine the frequencies of the vibrational modes of quantum number $\alpha$ (with progressively high energy $n=1,2, \ldots)$ are obtained from the relation

$$
\left[H, \Gamma_{\alpha}^{+}(n)\right]=\hbar \omega_{\alpha}(n) \Gamma_{\alpha}^{+}(n) .
$$

In the above equation $H$ is the total hamiltonian, sum of a single-particle and a two-body interaction term. In the present calculations we have used self-consistently the Skyrme plus pairing interaction given by the MSk7 parameter set. The RPA ground state energy is given by (cf. e.g. 11])

$$
E_{R P A}=E_{H F}-(2 \lambda+1) \sum_{\alpha, n} \hbar \omega_{\alpha}(n) \sum_{k i}\left|Y_{k i}^{\alpha}(n)\right|^{2},
$$

in keeping with the fact that the amplitudes $Y_{k i}^{\alpha}(n)$ are directly related to the ground state correlations induced by the corresponding vibrational modes. The second term of the r.h.s. is called correlation energy.

It is well known that open shell nuclei display a finite BCS gap $\Delta$, as a result of the pairing correlations acting between pairs of nucleons moving in time reversal states close to the Fermi energy. In closed shell nuclei, pairing correlations are not strong enough to overcome the large single-particle gap, and $\Delta=0$. Nevertheless, pairing plays an important role in determining the (lowlying) structure of these nuclei. In particular, it leads to pairing vibrations 9], which change in two the number of nucleons, that is vibrations which can be viewed as two correlated particles (pair addition modes) or two correlated holes (pair removal modes). An example of these modes is provided by the ground state of ${ }^{210} \mathrm{~Pb}$ and of ${ }^{206} \mathrm{~Pb}$ which can be viewed as the pair addition and the pair subtraction modes of ${ }^{208} \mathrm{~Pb}$ respectively. These modes are specifically probed through two particle transfer reactions [12]. The pair addition and pair subtraction modes can be written as

$$
\Gamma_{a}^{+}(n)=\sum_{k} X_{k}^{a}(n) \Gamma_{k}^{+}+\sum_{i} Y_{i}^{a}(n) \Gamma_{i}
$$

and

$$
\Gamma_{r}^{+}(n)=\sum_{i} X_{i}^{r}(n) \Gamma_{i}^{+}+\sum_{k} Y_{k}^{r}(n) \Gamma_{k},
$$

where $\Gamma_{k}^{+}=\left[a_{k}^{+} a_{k}^{+}\right]_{0}$ creates a pair of nucleons coupled to angular momentum zero in levels with energy larger than the Fermi energy $\left(\varepsilon_{k}>\varepsilon_{F}\right)$, while $\Gamma_{i}=\left[a_{i} a_{i}\right]_{0}$ annihilates a pair of nucleons in occupied states $\left(\varepsilon_{i} \leq \varepsilon_{F}\right)$. Use is made of a hamiltonian $H=H_{s p}+H_{p}$, sum of a single-particle term and of a pairing force with constant matrix $H_{p}=-G \sum_{j j^{\prime}} a_{j^{\prime}}^{+} a_{\tilde{j}^{\prime}}^{+} a_{\tilde{j}} a_{j}$, the commutation relation given in Eq.(3) leads to the dispersion relations

$$
\begin{aligned}
& \frac{1}{G}=\sum_{k} \frac{\Omega_{k}}{2 e_{k}-\hbar \omega_{a}(n)}+\sum_{i} \frac{\Omega_{i}}{2 e_{i}+\hbar \omega_{a}(n)}, \\
& \frac{1}{G}=\sum_{k} \frac{\Omega_{k}}{2 e_{k}+\hbar \omega_{r}(n)}+\sum_{i} \frac{\Omega_{i}}{2 e_{i}-\hbar \omega_{r}(n)},
\end{aligned}
$$

where $\Omega=(2 j+1) / 2$ is the pair degeneracy of the singleparticle orbital with total angular momentum $j$, while $e_{j}=\varepsilon_{j}-\varepsilon_{F}$. The amplitudes are

$$
X_{k}^{a}(n)=\frac{\Lambda_{a}(n) \sqrt{\Omega_{k}}}{2 e_{k}-\hbar \omega_{a}(n)}, \quad Y_{i}^{a}(n)=\frac{\Lambda_{a}(n) \sqrt{\Omega_{i}}}{2 e_{i}+\hbar \omega_{a}(n)},
$$

and

$$
X_{i}^{r}(n)=\frac{\Lambda_{r}(n) \sqrt{\Omega_{i}}}{2 e_{i}-\hbar \omega_{r}(n)}, \quad Y_{k}^{r}(n)=\frac{\Lambda_{r}(n) \sqrt{\Omega_{k}}}{2 e_{k}+\hbar \omega_{r}(n)},
$$

$\Lambda_{a}(n)$ and $\Lambda_{r}(n)$ being normalization constants determined from the relation given in Eq. (2).

In Fig. 1 we show the dispersion relations given in Eqs.(7) and (8) calculated for ${ }^{208} \mathrm{~Pb}$ for both protons and neutrons (cf. also [9]), making use of the valence orbitals of this nucleus. The valence orbitals used in our calculations were determined with the help of a WoodsSaxon potential with standard parametrization 15 and the energies have been replaced with the experimental values whenever available. The results obtained using the standard Woods-Saxon levels coincide with those calculated making use of the experimental energies within $2 \%$. Making use of the fact that the sum of the pairing binding energies of ${ }^{206} \mathrm{~Pb}$ and ${ }^{210} \mathrm{~Pb}$ as well as in ${ }^{206} \mathrm{Hg}$ and ${ }^{210} \mathrm{Po}$ are $\approx 2 \mathrm{MeV}$ (in this last case one has to take into account the Coulomb repulsion between the two protons, cf. e.g. [13]), one obtains the values of $2.7 \mathrm{MeV}$ and $2.2 \mathrm{MeV}$ for the neutron pair addition and pair removal energies, the corresponding values for the proton channel being $3.5 \mathrm{MeV}$ and $3.1 \mathrm{MeV}$ respectively (cf. refs [9], 13. and [14]). The associated $Y$ 's amplitudes are displayed in Tables [ and II]

Inserting these results in Eq.(4), one obtains the ground state correlation energy values $-0.399 \mathrm{MeV}$ (neutrons) and - $-0.449 \mathrm{MeV}$ (protons) respectively. In all calculations we have kept the contribution of only the lowest $(n=1)$ pair addition and pair subtraction modes, 

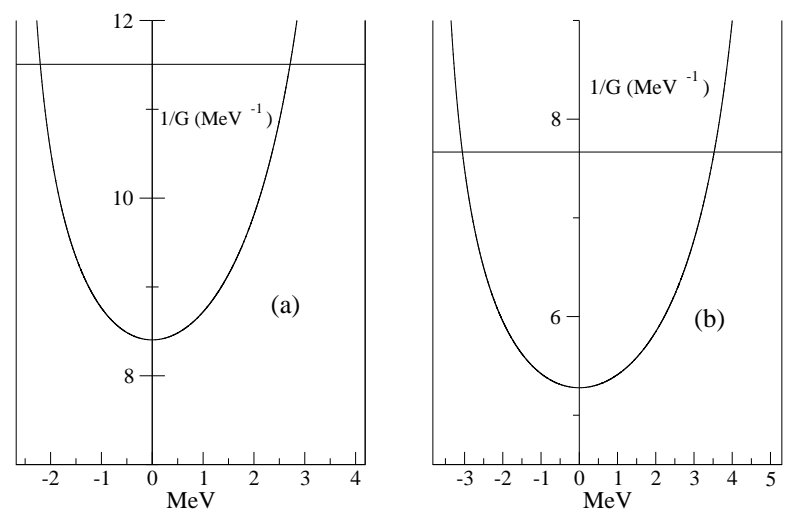

FIG. 1: Monopole-pairing-vibrations dispersion relation for (a) neutrons and (b) protons for the nucleus ${ }^{208} \mathrm{~Pb}$

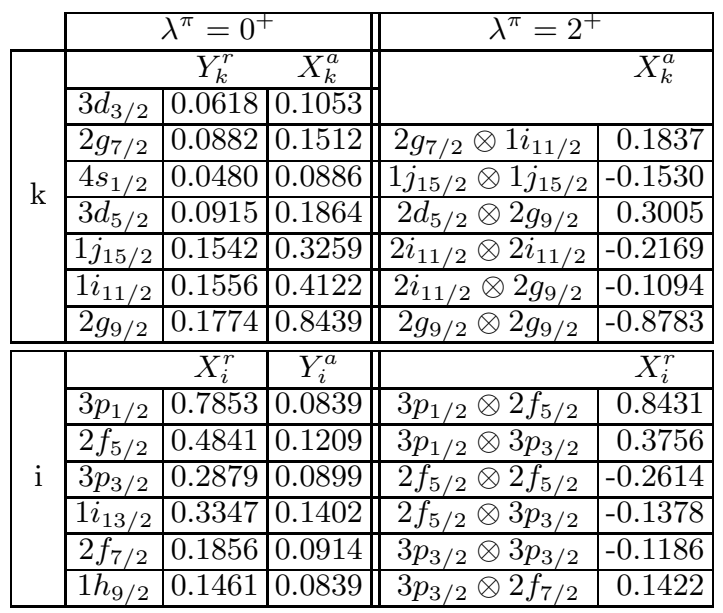

TABLE I: RPA wavefunctions of the neutron pair addition (a) and pair removal (r) modes of ${ }^{208} \mathrm{~Pb}$ with multipolarities and parity $\lambda^{\pi}=0^{+}, 2^{+}$.

in keeping with the fact that, as a rule, the $n \neq 1$ modes are much less collective. Pairing vibrations with multipolarity $\lambda \neq 0$, in particular quadrupole and hexadecapole pairing vibrations, have also been identified around closed shell nuclei (cf. [12], 13], 14] and refs therein). In Table III we display the contributions to the ground state energy (i.e. $E_{R P A}$ as defined in Eq.(4A) associated with the monopole, quadrupole and hexadecapole pair addition and pair removal modes for both neutrons and protons associated with ${ }^{208} \mathrm{~Pb}$, the summed contribution amounting to $-1.981 \mathrm{MeV}(\approx-1.196 \mathrm{MeV}$ $-0.785 \mathrm{MeV})$.

In Table IV we collect the corresponding contribution for a number of $\mathrm{Pb}$-isotopes. Because pairing vibrations are collective modes only around closed shell nuclei, where particles and holes can be clearly distinguished, becoming non-collective two-quasiparticle modes outside closed shells (cf. e.g. ref. [5]) we have considered the contribution of neutron pairing vibrations only for the closed shell system (while the proton pairing vibration were taken into account for all isotopes). Also shown

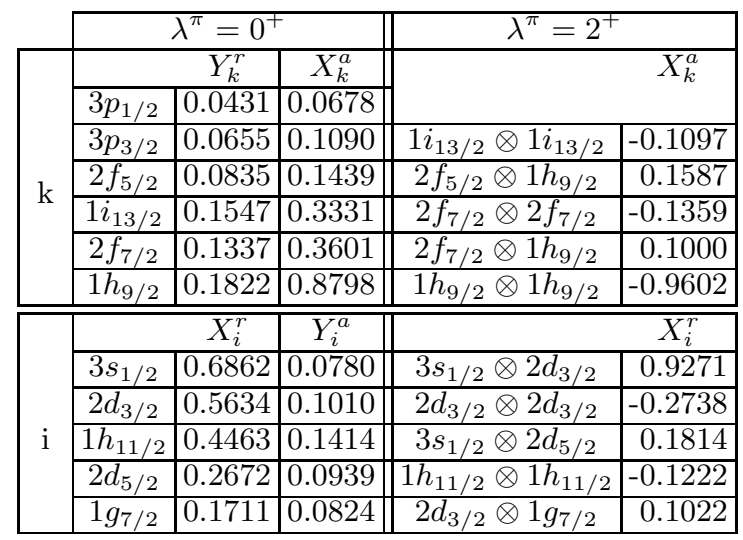

TABLE II: RPA wavefunctions of the proton pair addition (a) and pair removal (r) modes of ${ }^{208} \mathrm{~Pb}$ with multipolarities and parity $\lambda^{\pi}=0^{+}, 2^{+}$.

\begin{tabular}{|c|c|c|c|c|c|}
\hline \multicolumn{2}{|c|}{$0^{+}$} & \multicolumn{2}{c|}{$2^{+}$} & \multicolumn{2}{c|}{$4^{+}$} \\
\hline $\mathrm{n}$ & $\mathrm{p}$ & $\mathrm{n}$ & $\mathrm{p}$ & $\mathrm{n}$ & $\mathrm{p}$ \\
\hline-0.399 & -0.449 & -0.609 & -0.244 & -0.189 & -0.092 \\
\hline
\end{tabular}

TABLE III: Ground state correlation energies, arizing from the neutron (n) and protons ( $\mathrm{p}$ ) monopole, quadrupole and hexadecapole pairing vibrations in ${ }^{208} \mathrm{~Pb}$.

\begin{tabular}{|c|r|r|r|r|r|}
\hline & ${ }^{204} \mathrm{~Pb}$ & ${ }^{206} \mathrm{~Pb}$ & ${ }^{208} \mathrm{~Pb}$ & ${ }^{210} \mathrm{~Pb}$ & ${ }^{212} \mathrm{~Pb}$ \\
\hline$p$-h vibrations & -2.793 & -2.709 & -2.237 & -2.801 & -3.173 \\
\hline pairing vibrations & -0.785 & -0.785 & -1.981 & -0.785 & -0.785 \\
\hline
\end{tabular}

TABLE IV: Ground state correlation energies for the $\mathrm{Pb}-$ isotopes.

\begin{tabular}{|c|c|c|c|c|c|}
\hline & ${ }^{40} \mathrm{Ca}$ & ${ }^{42} \mathrm{Ca}$ & ${ }^{44} \mathrm{Ca}$ & ${ }^{46} \mathrm{Ca}$ & ${ }^{48} \mathrm{Ca}$ \\
\hline$p$ - $\mathrm{h}$ vibrations & -0.886 & -1.418 & -1.606 & -1.391 & -0.547 \\
\hline pairing vibrations & -4.761 & -2.978 & -3.239 & -3.500 & -5.823 \\
\hline
\end{tabular}

TABLE V: Ground state correlation energies for the Caisotopes.

\begin{tabular}{|c|c|c|}
\hline & \multicolumn{2}{|c|}{$\sigma(\mathrm{MeV})$} \\
\hline & ref. [4] & ref. [4] + RPA \\
\hline $\mathrm{Pb}$ & 0.646 & 0.543 \\
$\mathrm{Ca}$ & 1.200 & 0.466 \\
\hline $\bar{\sigma}$ & 0.964 & 0.505 \\
\hline
\end{tabular}

TABLE VI: Root mean square error associate with the HFBCS MSk7 mass formula of ref. [4] and to this formula (with sligthly adjusted parameters) plus the correlation contributions associated with surface and pairing vibrations calculated in the RPA. The quantity $\bar{\sigma}=\left(\frac{\sigma_{C a}^{2}+\sigma_{P b}^{2}}{2}\right)^{1 / 2}$ is shown in the last line.

in table IV are the contribution to $E_{R P A}$ arising from the low-lying collective particle-hole vibrations calculated making use of the MSk7 interaction to determine the single particle states and the particle-hole correlated modes (cf. Eq.(1)). Quadrupole and octupole vibrations with energy $<7 \mathrm{MeV}$, and exausting $\geq 2 \%$ of the non-energy 
weighted sum rule were included in the calculation of $E_{R P A}$. These conditions essentially select the lowest (one-two) states displaying correlated wavefunctions.

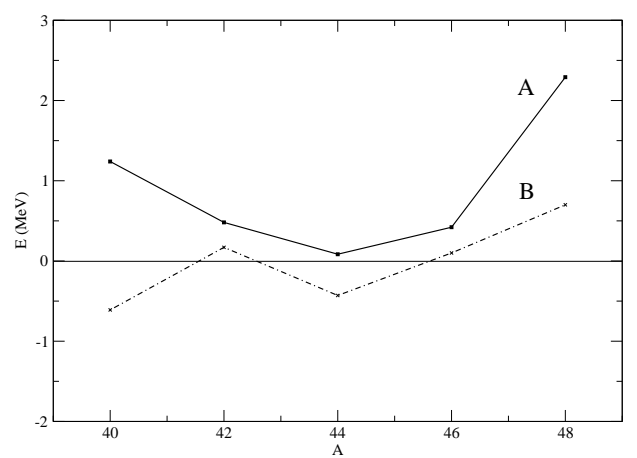

FIG. 2: Difference between the calculated and the experimental ground state energies. The curve (A) refers to the HFBCS MSk7 results of ref. [4]. The curve (B) was obtained adding $p$ - $h$ and pairing correlations to the curve (A) and renormalizing the Skyrme parameters as indicated in the text.

Similar calculations were repeated for the calcium isotopes ${ }^{40-48} \mathrm{Ca}$. In this case, the contribution of the proton pairing vibrations calculated for the closed shell systems ${ }^{40} \mathrm{Ca}$ and ${ }^{48} \mathrm{Ca}$ were linearly interpolated for the other isotopes, and no hexadecapole modes were considered. In Table $\nabla$ we show the corresponding results, together with the contribution of the particle-hole vibrational modes. When adding the results of Tables IV and
D] to the HFBCS MSk7 mass formula of Ref. [4], the parameters of the Skyrme interaction should be refitted in order to provide the best reproduction of experimental masses. This should be done on a large sample of isotopes and it is beyond the purpose of the present paper. If we restrict ourselves to Ca-isotopes ( $\mathrm{Pb}$-isotopes), a slight renormalization by a factor 0.9964 (0.99945) of the Skyrme parameters $t_{i}, W_{0}$ is enough to shift the calculated value of the masses upwards by $\approx 4.3 \mathrm{MeV}(3.5$ $\mathrm{MeV}$ ) and minimize the rms deviation, giving the results displayed in Table VI and, for the Calcium isotopes, also shown in Fig. 2 Averaging the rms deviations associated with $\mathrm{Ca}$ - and $\mathrm{Pb}$-isotopes, leads to a value of 0.505 $\mathrm{MeV}$ as compared to the value of $0.964 \mathrm{MeV}$ obtained making use of the results of ref. 3]. Although a global readjustment of the mean field parameters should be envisaged, the fact that the locally extracted rms deviations have been reduced by a factor of $\approx 2$ can be considered meaningful.

Similarly to what was observed in assessing the role played by zero point fluctuations associated with surface and pairing vibrations in connection with the nuclear mean square radius [16, 17, 18], the alignment of rotational nuclei [19] and the pairing phase transition as a function of angular momentum [20, 21, 22], we conclude that zero point fluctuations play an important role in providing the A-dependent contributions to nuclear masses needed to make mean field theory, including its time dependence, a quantitative predictive tool.
[1] When neutrons are progressively added to a normal nucleus, the Pauli principle forces them into states of higher momentum. When the core becomes neutron saturated, the nucleus expels most of the wavefunction of the last neutrons outside, to region of lower density, which because of its larger size can have lower momentum. In most cases neutrons essentially drip off from the nucleus, defining the lines of stability for neutron number in the chart of nuclides (i.e. the neutron drip line for which the neutron binding energy is equal to zero).

[2] C.E.Rolfs and W.S.Rodney, Cauldrons in the cosmos, The University of Chicago Press, Chicago (1988)

[3] S.Goriely, M.Samyn, P.H.Heenen, J.M.Pearson and F.Tondeur, Phys. Rev. C 66 (2002) 024326-1

[4] S.Goriely, F.Tondeur and J.M.Pearson, At. Data Nucl. Data Tables $7 \mathbf{7}$ (2001) 311.

[5] I. Tanihata, J. Phys. G22 (1996) 157.

[6] G.F. Bertsch and K. Hagino, Phys. Atom. Nucl. 64(2001) 533

[7] I. Stetcu and C.W.Johnson, Phys. Rev. C 66 (2002) 034301.

[8] M.Bender, G.F.Bertsch, P.-H.Heenen, nucl-th/0311026

[9] D.R.Bes and R.A.Broglia, Nucl. Phys. 80 (1966) 289

[10] P.W.Anderson, Phys. Rev. 112 (1958) 1900
[11] P.Ring and P.Schuck, The nuclear many-body problem, Springer Heidelberg (1980)

[12] R.A.Broglia, O.Hansen and C.Riedel, Advances in Nuclear Physics, Ed. M. Baranger and E. Vogt, Plenum Press, Vol.6, 287 (1973)

[13] P.F.Bortignon, R.A.Broglia, D.R.Bes and R.Liotta, Phys.Rep. 30C (1977) 305

[14] D.R.Bes and R.A.Broglia, Phys. Rev. C 6 (1971) 2349

[15] A.Bohr and B.R.Mottelson, Nuclear Structure, Vol. I, Benjamin, New York (1969)

[16] H. Esbensen and G.F. Bertsch, Phys. Rev. C28 (1983) 355

[17] F. Barranco and R.A.Broglia, Phys. Lett. 151B (1985) 90

[18] F. Barranco and R.A.Broglia, Phys. Rev. Lett. 59 (1987) 2724

[19] F. Barranco, M. Gallardo and R.A.Broglia, Phys. Lett. 198B (1987) 19

[20] R.A.Broglia, C.Riedel and B.Sørensen, Nucl. Phys. A115 (1968) 273

[21] Y. R. Shimizu, J. Garrett, R.A.Broglia, M. Gallardo and E. Vigezzi, Rev. of Mod. Phys. 61 (1989) 131

[22] R.S. Nikam and P. Ring, Phys. Rev. Lett. 58(1987)980 